\title{
Exact diffraction calculation from fields specified over arbitrary curved surfaces
}

\author{
G. Bora Esmer *, Levent Onural, Haldun M. Ozaktas \\ Bilkent University, Department of Electrical and Electronics Engineering, TR-06800 Bilkent, Ankara, Turkey
}

\section{A R T I C L E I N F O}

Article history:

Received 23 December 2010

Received in revised form 6 July 2011

Accepted 11 July 2011

Available online 31 July 2011

\section{Keywords:}

Scalar optical diffraction

Plane wave decomposition

Signal decomposition

Eigenvalue distribution

\begin{abstract}
A B S T R A C T
Calculation of the scalar diffraction field over the entire space from a given field over a surface is an important problem in computer generated holography. A straightforward approach to compute the diffraction field from field samples given on a surface is to superpose the emanated fields from each such sample. In this approach, possible mutual interactions between the fields at these samples are omitted and the calculated field may be significantly in error. In the proposed diffraction calculation algorithm, mutual interactions are taken into consideration, and thus the exact diffraction field can be calculated. The algorithm is based on posing the problem as the inverse of a problem whose formulation is straightforward. The problem is then solved by a signal decomposition approach. The computational cost of the proposed method is high, but it yields the exact scalar diffraction field over the entire space from the data on a surface.
\end{abstract}

(C) 2011 Published by Elsevier B.V.

\section{Introduction}

When the input field is specified over a planar surface, the calculation of monochromatic scalar optical diffraction can be accomplished in a straightforward manner by plane wave decomposition or the Rayleigh-Sommerfeld diffraction integral, or by other methods derived from these. Integration over the planar surface allows computation of the exact diffraction field over the entire space. However, if the input field is specified over a curved surface, rather than a planar surface, straightforward integration over the curved surface may not provide the exact field over the entire space. Calculation of the exact diffraction field from a curved surface requires greater care and is the subject of this work [16].

Diffraction field calculation by direct integration over the surface on which the input field is specified, is essentially a weighted superposition of the free-space diffraction kernel. However, direct integration gives the exact field only when the integrated surface field value remains unaltered by the propagation from other surface elements. If we simply ignore such mutual interactions, the calculated field will be different from the actual field. The method we set forth is based on the following observation. No such interactions exists when the input field is specified over a plane; therefore it is straightforward to express the field on an arbitrary curved surface (and indeed any region of the entire space) as a weighted superposition integral of the free-space diffraction kernel over a planar surface. In the problem we wish to solve, the field is known over a curved surface and we wish to obtain the field over a planar surface (which would then also enable us to calculate it over the entire space). Since it is not straightforward

\footnotetext{
* Corresponding author.

E-mail address: borahan@ee.bilkent.edu.tr (G.B. Esmer).
}

to express the field on the planar surface in terms of the field on the curved surface, we express the field on the curved surface in terms of that on the planar surface, and solve an inverse problem to obtain the field on the planar surface. The inverse problem arising from this exact formulation can be solved by employing several methods and standard algorithms, each with their pros and cons. In this paper, we propose a signal decomposition algorithm for this purpose.

Our interest in diffraction calculations from curved surfaces stems from our work on computer generated holography (CGH) and threedimensional imaging and television [1-4,11,17,20-23]. Since the diffraction field from an arbitrarily shaped object is the field that we desire to recreate at the display end, its accurate calculation is of utmost importance.

In both computer graphics and $\mathrm{CGH}$, objects are commonly modeled as a set of sample points distributed over space $[8,9,14,15]$. It is assumed that the characteristics of the continuous object can be sufficiently represented by these sample points. A straightforward approach to compute the diffraction field created by an object is to superpose the fields created by each sample point of the object; doing so amounts to treating each sample point as a light source. We will refer to diffraction field calculation approaches based on superposition of the fields at each sample point of the object as "source model" approaches. In these approaches, it is assumed that the value of each source is independent of the field at other points. Then, the independently computed fields from these points are superposed. The calculated field will be the same as the actual field only if the points truly act as sources (i.e., if the values of these sources are not perturbed by the superposed field generated by the other sources). However, usually there are complicating interactions. Consequently, the field calculated using the source model will not be exact or may even be significantly in error. Diffraction field calculations based on the source model have the advantage of having reasonable 
computational complexities, but they are not necessarily exact except when all the sample points are given over a planar surface.

With the term mutual interaction we refer to the fact that the field at a given input point is not independent of the field at the other input points; in other words, it is not possible to specify them independently and arbitrarily.

Ignoring the mutual interactions and straightforwardly superposing the specified input field values will not give exact results. Instead, a simultaneous calculation of the diffraction field due to the given input points is necessary. We will refer to approaches based on such simultaneous calculation of the diffraction field as "field model" approaches. The diffraction field computation method presented in this paper is based on such an approach and uses a decomposition of the field specified over an orientable manifold onto a function set obtained from the intersection of the propagating plane waves by the manifold.

The algorithm we propose can be used for both two-dimensional (2D) and three-dimensional (3D) spaces. For simplicity we will first discuss the 2D case. In the 3D case, numerical issues due to larger data sets arise. Nevertheless, as a proof of concept the extension of the proposed algorithm to the 3D case is also presented.

\section{Calculation of the diffraction field using the source model}

Computation of the diffraction field arising from the samples of an object or a set of given sample points over the space can be performed in several ways. One of the most commonly employed methods is to superpose the fields emitted by the sample points. As discussed in Section 1, we refer to such methods as source model methods. In the literature, there are several diffraction field computation algorithms based on the source model approach $[5,8,9,13]$. Implementation of source model algorithms is rather straightforward because mutual interactions are not taken into consideration.

Depending on the distribution of the sample points over the space, the effect of mutual interactions can be significant. A simple example will help illustrate the issues involved (Fig. 1). We consider three points $P_{1}, P_{2}, P_{3}$ which create the field, $\psi(\mathbf{x})$, over the entire space. Here the coordinate vector $\mathbf{x}$ denotes $\left[\begin{array}{ll}x & z\end{array}\right]^{T}$. According to the source model approach, the field over the reference line is computed by superposing the fields created by the field samples at $P_{1}=\mathbf{x}_{1}, P_{2}=\mathbf{x}_{2}$, $P_{3}=\mathbf{x}_{3}$ (which are assumed as sources). The diffraction field

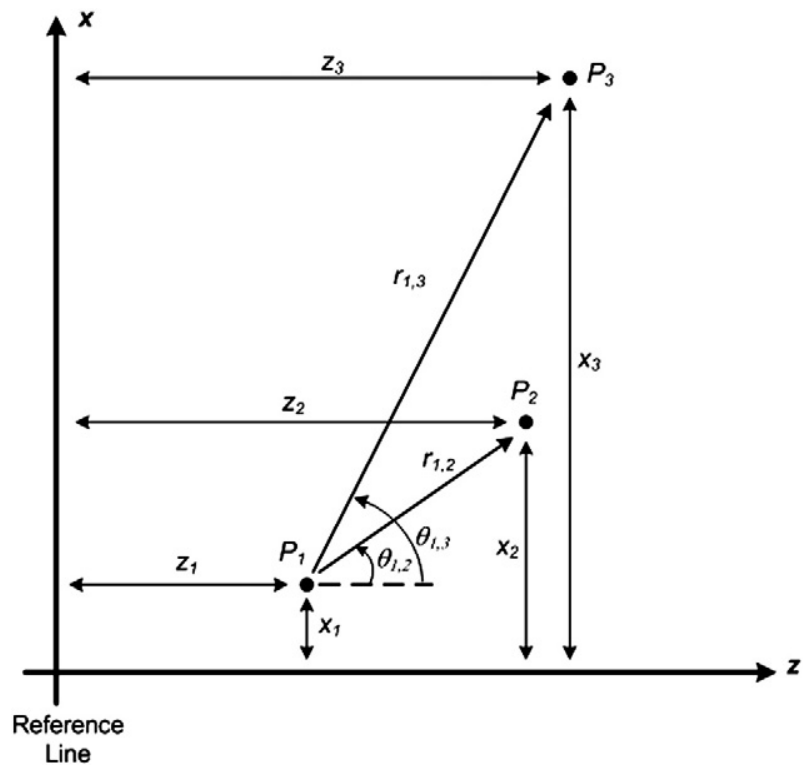

Fig. 1. Illustration of possible mutual couplings in the source model approach for a $2 \mathrm{D}$ space. The $x$-axis is taken as the reference line. emanating from these sample points is usually calculated by using the kernel of the Rayleigh-Sommerfeld (RS) diffraction integral. More specifically, the field $f(\mathbf{x})$ over the line $z=0$ is expressed in terms of the strengths $\psi\left(\mathbf{x}_{1}\right), \psi\left(\mathbf{x}_{2}\right), \psi\left(\mathbf{x}_{3}\right)$ of the sources as

$f(\mathbf{x})=\psi\left(\mathbf{x}_{1}\right) h\left(\mathbf{x}-\mathbf{x}_{1}\right)+\psi\left(\mathbf{x}_{2}\right) h\left(\mathbf{x}-\mathbf{x}_{2}\right)+\psi\left(\mathbf{x}_{3}\right) h\left(\mathbf{x}-\mathbf{x}_{3}\right)$

where $h(\mathbf{x})$ denotes the $2 \mathrm{D}$ version (i.e., $y=0$ case) of the RS diffraction kernel due to propagating waves,

$h(\mathbf{x})=\frac{1}{j \lambda} \frac{\exp \left(j \frac{2 \pi}{\lambda} \sqrt{x^{2}+y^{2}+z^{2}}\right)}{\sqrt{x^{2}+y^{2}+z^{2}}} \cos \theta$,

where $\cos \theta=\frac{z}{\sqrt{x^{2}+y^{2}+z^{2}}}$ and $\lambda$ is the optical wavelength. The above expression gives the field on the reference plane arising from these three points. Quite often, the $\cos \theta$ term is ignored; omitting this term may result in significant errors if $\theta$ is not small. It is instructive to note that the Rayleigh-Sommerfeld kernel is the impulse response for field computations where the wave propagates out from a planar surface; this is different than a spherically symmetric propagation out from a simple free-standing point source. Using the expression we have obtained for the field on the reference line, we may now calculate back the field values at $P_{1}, P_{2}, P_{3}$ again by using the RS diffraction integral. In general it turns out that the values obtained are not equal to the original values specified at $P_{1}, P_{2}, P_{3}$. Thus even with such a simple scenario, it is possible to see the effect of the interactions between the specified source points. For instance, the deviation between the calculated field and the initially specified field at $P_{1}$ is found as

$\Delta \psi\left(\mathbf{x}_{1}\right)=\psi\left(\mathbf{x}_{2}\right) \frac{\cos \theta_{1,2}}{\left|\mathbf{r}_{1,2}\right|} \exp \left(j \frac{2 \pi}{\lambda}\left|\mathbf{r}_{1,2}\right|\right)+\psi\left(\mathbf{x}_{3}\right) \frac{\cos \theta_{1,3}}{\left|\mathbf{r}_{1,3}\right|} \exp \left(j \frac{2 \pi}{\lambda}\left|\mathbf{r}_{1,3}\right|\right)$

where $\mathbf{r}_{1,2}$ is the vector between points $P_{1}$ and $P_{2}$, and $\theta_{1,2}$ denotes the angle between the vector $\mathbf{r}_{1,2}$ and the $z$-axis as shown in Fig. 1. Similarly, the vector $\mathbf{r}_{1,3}$ denotes the difference between the position vectors $\mathbf{x}_{1}$ and $\mathbf{x}_{3}$, and $\theta_{1,3}$ is the angle between the vector $\mathbf{r}_{1,3}$ and the $z$-axis. This deviation is exactly the additional field imposed on $P_{1}$ by the sources at $P_{2}$ and $P_{3}$ under the RS model. A similar deviation can be shown also for $P_{2}$ or $P_{3}$. These deviations from the initially specified fields at each sample point depend on the initial field values on the other sample points and their mutual positions in space. As a result of these interactions between the fields emanating from the sources, the source model approach may not provide the exact field over the entire space.

For a discrete set of points, there will be no mutual interactions among the sample points if the following condition is satisfied:

$\psi\left(\mathbf{x}_{i}\right)=\sum_{\substack{\mathrm{j} \\ \mathrm{j} \neq \mathrm{i}}} \psi\left(\mathbf{x}_{\mathrm{j}}\right) h\left(\mathbf{x}_{\mathrm{i}}-\mathbf{x}_{\mathrm{j}}\right)=0, \forall \mathrm{i}$

where $\psi\left(\mathbf{x}_{\mathrm{j}}\right) h\left(\mathbf{x}_{\mathrm{i}}-\mathbf{x}_{\mathrm{j}}\right)$ is the field generated at location $\mathbf{x}_{\mathrm{i}}$ by point $\mathrm{j}$. This is satisfied for the classical case of diffraction computation from the points which lie on a plane.

By the way, above observations are still valid if other diffraction models, (for example, the Fresnel-Kirchoff approximation) are utilized instead of the RS formulation.

In the source model, the computation of the continuous diffraction field over the entire space can be expressed as an integral over a surface $S_{a}$ as

$\psi(\mathbf{x})=\int_{S_{a}} \psi(\mathbf{x}) h(\mathbf{x}-\mathbf{x}) d S$ 
where $S_{a}$ denotes a curved surface, the vector $\mathbf{x}=[x, y, z]^{\mathrm{T}}$ is used to indicate the position of the calculated diffraction field, and $\mathbf{x}=\left[x^{\prime}, y^{\prime}, z^{\prime}\right]^{\mathrm{T}}$ is another position vector running over the surface of $S_{a}$. The kernel of the integral, $h(\mathbf{x})$, is as defined in Eq. (2) when the RS kernel is used. For computational purposes, the above integral must be discretized by sampling the field on the surface $S_{a}$. Then, the diffraction field over the space is obtained as the superposition of the fields from the sample points. Each emanating field is expressed by a column vector $\mathbf{h}\left(\mathbf{x}_{\mathrm{i}}\right)$, which is obtained by sampling the RS diffraction integral kernel defined in Eq. (2). (If propagation along the reverse direction with respect to $z$ axis is involved, then the conjugate of $\mathbf{h}\left(\mathbf{x}_{\mathbf{i}}\right)$ should be employed.) Thus the source model employed in our simulations can be expressed as [4]

$\mathbf{f}=\sum_{i=1}^{s} \psi\left(\mathbf{x}_{\mathrm{i}}\right) \mathbf{h}^{*}\left(\mathbf{x}_{\mathrm{i}}\right)$

where $s$ is the number of samples in space, and $\psi\left(\mathbf{x}_{\mathrm{i}}\right)$ denotes the complex amplitude of the $i$ th field sample. Propagation from the reference line towards the sample point, and propagation from the sample point back to the reference line are related to each other by a reversal of the sign of $z_{\mathrm{i}}$ (i.e., propagation along $z$ or $-z$ directions). In the source model, each field sample is considered as a source. The locations of the sample points along the $x, y, z$ axes are denoted as $x_{i}, y_{i}$, $z_{i}$, respectively. The column vector $\mathbf{h}^{*}\left(\mathbf{x}_{\mathbf{i}}\right)$ denotes the field propagating from a point source at $\left(x_{i}, y_{i}, z_{i}\right)$ towards the reference line at $z=0$.

\section{Computation of the diffraction field using the field model}

Computation of the diffraction field by using the source model may not always yield sufficiently accurate results as a consequence of the mutual interactions between the specified sample points over a curved surface. In the field model approach, such mutual interactions are taken into consideration by enabling the calculation of the exact field over the entire space. There is no mutual interaction between the sample points when they lie on a planar surface and the RS model is employed where $\theta$ is measured from the surface normal. Then, the cosine term in Eq. (2) becomes zero, because $\theta= \pm \frac{\pi}{2}$. Therefore, while it is not straightforward to express the field on a planar surface in terms of that on a curved surface, it is straightforward to express the field on a curved surface in terms of that on a planar surface. Thus we can easily write an expression for the inverse of the problem we wish to solve. Subsequent inversion will then allows us to obtain the exact field. The following sections detail and illustrate this approach.

\section{Mathematical basis of the proposed field model algorithm}

Propagating scalar monochromatic waves have to satisfy the wave equation. One of the simplest sets of waves that satisfy the wave equation is the set of propagating monochromatic plane waves $[6,7,12]$. It is well known that there is a Fourier transform relationship between transverse profiles of the diffraction field and the complex coefficients of the propagating plane wave components $[6,7,10$ $12,18,19]$.

For simplicity we will first consider the 2D case, where we wish to obtain the 2D field arising from the field specified on a curved line on that $2 \mathrm{D}$ space. The diffraction field over the entire $2 \mathrm{D}$ space can be expressed in terms of propagating plane waves as,

$\psi(\mathbf{x})=\int_{k_{x} \in K} A_{1 \mathrm{D}}\left(k_{x}\right) \exp \left(j \mathbf{k}^{\mathrm{T}} \mathbf{x}\right) d k_{x}$,

where $K$ is the spatial bandwidth of the field and $A_{1 \mathrm{D}}\left(k_{x}\right) d k_{x}$ denotes the complex amplitudes of the propagating plane waves along the direction indicated by $\mathbf{k}$. The position over $2 \mathrm{D}$ space is denoted by $\mathbf{x}=$ $[x, z]^{\mathrm{T}}$. The spatial frequencies along $x$ and $z$ axes are represented by the vector $\mathbf{k}=\left[k_{x}, k_{z}\right]$ where $k_{x}$ is the spatial frequency along the $x$ axis and $k_{z}$ is the spatial frequency along the $z$ axis. Since we are dealing with monochromatic waves, the spatial frequency $k_{z}$ is a function of $k_{x}$,

$k_{z}=\sqrt{k^{2}-k_{x}^{2}}$

where $k$ is the wave number and is equal to $\frac{2 \pi}{\lambda}$.

The transversal line $z=0$ will be denoted by $S_{0}$. The field on this line is a 1D profile of the 2D diffraction field over the entire 2D space. Each propagating plane wave corresponds to a complex harmonic over $S_{0}$, and these complex harmonics are orthogonal to each other. Therefore, $A_{1 D}\left(k_{x}\right)$ can be found by performing,

$$
\begin{aligned}
\left.\left\langle\psi(\mathbf{x}), \exp \left(j \mathbf{k}^{\mathrm{T}} \mathbf{x}\right)\right\rangle\right|_{S 0} & =\int_{S_{0}} \psi(\mathbf{x}) \exp \left(-j \mathbf{k}^{\mathrm{T}} \mathbf{x}\right) d x \\
& =2 \pi A_{1 \mathrm{D}}\left(k_{x}\right)
\end{aligned}
$$

where $\langle\cdot, \cdot\rangle$ denotes the inner product. Unfortunately, a similar approach can not be used for fields specified over a curved surface $S_{a}$. The profiles of the propagating plane waves over the curved $S_{a}$ may not be orthogonal to each other even if the field specified on $S_{a}$ can be expressed as a linear superposition of such profiles. With "profile" we mean the part of the 2D field obtained by intersecting it with the surface $S_{a}$. The non-orthogonality of the profiles of plane waves on curved surfaces is illustrated by a simple example given in the Appendix A.

As mentioned above, the diffraction field over the entire space can be expressed as a superposition of propagating plane waves. Therefore, one approach to calculate the diffraction field over the entire 2D space is to find the complex amplitudes of the plane waves, from the field on the curved line. The algorithm we propose is based on the relation between the diffraction field on $S_{a}$ and the complex amplitudes of the propagating plane waves. In the proposed algorithm, the diffraction field over the entire space is expressed as

$\hat{\psi}(\mathbf{x})=\int_{k_{x} \in K} \hat{A}_{1 \mathrm{D}}\left(k_{x}\right) \exp \left(j \mathbf{k}^{\mathrm{T}} \mathbf{x}\right) d k_{x}$,

where

$\hat{A}_{1 \mathrm{D}}\left(k_{x}\right)=\underset{A_{1 \mathrm{D}}\left(k_{x}\right)}{\arg \min }\left\{\int_{S_{a}}\left|\psi(\mathbf{x})-\int_{k_{x} \in K} A_{1 \mathrm{D}}\left(k_{x}\right) \exp \left(j \mathbf{k}^{\mathbf{T}} \mathbf{x}\right) d k_{x}\right|^{2} d S\right\}$.

$\hat{A_{1 D}} \hat{\left(k_{x}\right)}$ denotes our estimate of the complex amplitudes of the plane waves that minimize the difference between the estimated field and the given field over $S_{a} . \hat{\psi}(\mathbf{x})$ is the obtained field using the estimated $\hat{A}_{1 \mathrm{D}}\left(k_{x}\right)$. By the way, since the original 2D field $\psi(\mathbf{x})$ was a pattern that can be obtained by propagating plane waves, the estimation error will be zero. Yet, to reduce the computational complexities, we introduce further restrictions: we specify that the diffraction field over the entire space consists of a finite number of plane waves:

$\psi(\mathbf{x})=\sum_{k_{M}} A_{1 \mathrm{D}}\left(k_{m_{x}}\right) \exp \left(j \mathbf{k}_{M}^{T} \mathbf{x}\right)$

where $k_{M}=\left[k_{m_{x}}, k_{m_{z}}\right]^{\mathrm{T}}$. The relation between $k_{m_{x}}$ and $k_{m_{z}}$ is the same as that is given by Eq. (8): $k_{m_{z}}=\sqrt{k^{2}-k_{m_{x}}^{2}}$. The spatial frequency $k_{m_{x}}=\frac{2 \pi}{M X} m_{x}$ where $m_{x} \in[-M / 2, M / 2)$. Then, the least-squares problem given by Eq. (11) can be expressed as

$\hat{A}_{1 \mathrm{D}}\left(k_{m_{x}}\right)=\underset{A_{1 \mathrm{D}}\left(k_{m_{x}}\right)}{\arg \min }\left\{\int_{S_{a}}\left|\psi(\mathbf{x})-\sum_{\mathbf{k}_{M}} A_{1 \mathrm{D}}\left(k_{m_{x}}\right) \exp \left(j \mathbf{k}_{M}^{T} \mathbf{x}\right)\right|^{2} d S\right\}$. 
The estimate of the diffraction field over $S_{a}$ is related to the estimates of the complex plane-wave coefficients as

$\hat{\psi}(\mathbf{x})=\sum_{\mathbf{k}_{M}} \hat{A}_{1 \mathrm{D}}\left(k_{m_{x}}\right) \exp \left(j \mathbf{k}_{M}^{T} \mathbf{x}\right)$.

To estimate the complex amplitudes of the plane waves, we use the profiles of the plane waves on $S_{a}$, but as already noted these functions may not be orthogonal. To obtain orthogonal functions, we employ Gram-Schmidt orthogonalization, and then these obtained orthogonal functions are used to decompose the field on $S_{a}$.

The expression given in Eq. (12) can be written in more compact form using matrix-vector notation as

$\psi=\mathbf{H a}$

where $\psi$ is a vector that represents the field on the points located on $S_{a}$ and the vector a denotes the complex amplitudes of the plane waves that we wish to obtain. The system matrix $\mathbf{H}$ is formed by the columns from the profiles of the plane waves on $S_{a}$, as,

$$
\begin{aligned}
\mathbf{H} & =\left.\left[\exp \left(j \mathbf{k}_{1}^{T} \mathbf{x}\right)\left|\exp \left(j \mathbf{k}_{2}^{T} \mathbf{x}\right)\right| \mid \exp \left(j \mathbf{k}_{M}^{T} \mathbf{x}\right)\right]\right|_{\mathbf{x} \in S_{a}} \\
& =\left[\boldsymbol{\Phi}_{1}\left|\Phi_{2}\right| \ldots \mid \Phi_{M}\right] .
\end{aligned}
$$

The matrix $\mathbf{H}$ has $M$ column vectors, denoted by $\boldsymbol{\phi}_{i}$. Following orthogonalization of the functions over the curved surface using the Gram-Schmidt procedure, Eq. (15) can be expressed as

$\boldsymbol{\psi}=\mathbf{Q R a}$

where the columns of the matrix $\mathbf{Q}$ form the orthogonal basis functions that describe the system. The matrix $\mathbf{Q}$ has $M$ column vectors:

$\mathbf{Q}=\left[\mathbf{q}_{1}\left|\mathbf{q}_{2}\right| \ldots \mid \mathbf{q}_{M}\right]$.

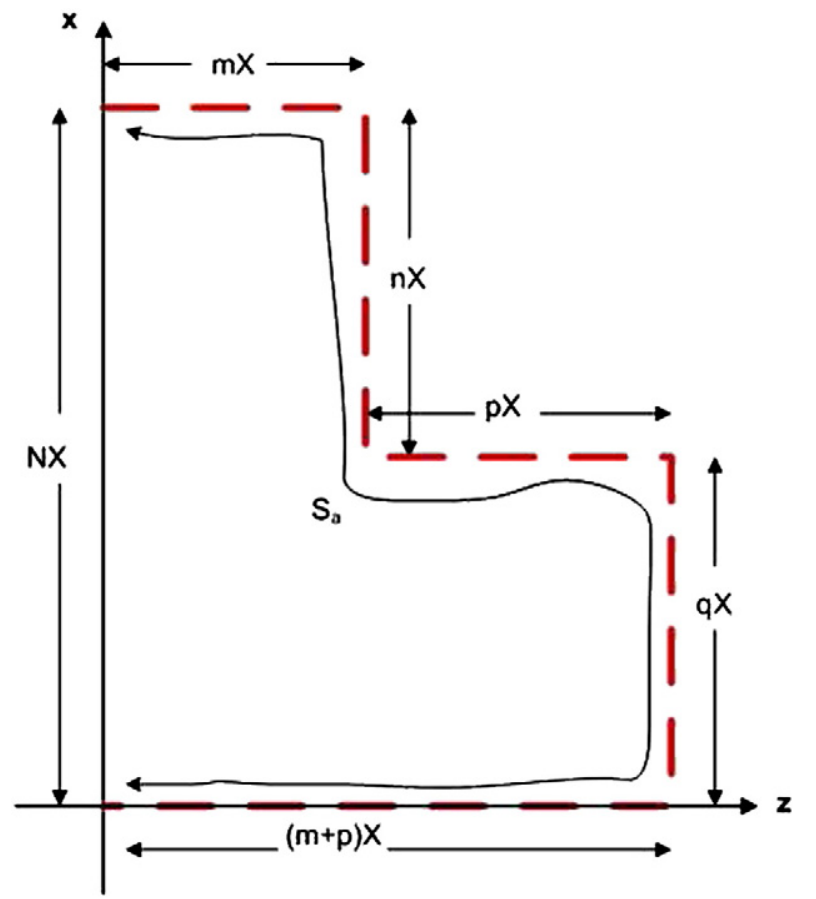

Fig. 2. Example where we wish to reconstruct the $2 \mathrm{D}$ diffraction field from the field given over the 1D curved surface $S_{a}$ which is a union of line segments as denoted by the thick dashed lines. The integers $N, m, n, p, q$ denote the number of samples spaced by $X$ along the corresponding segments.

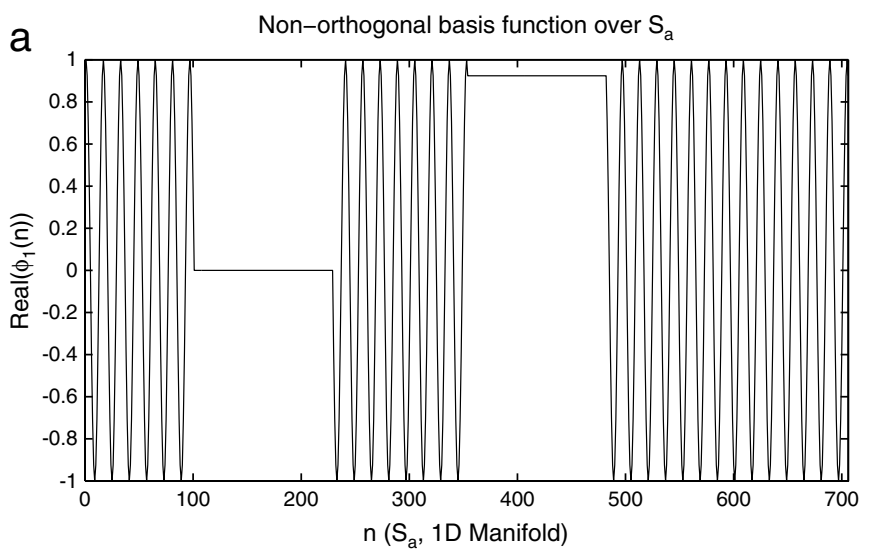

b

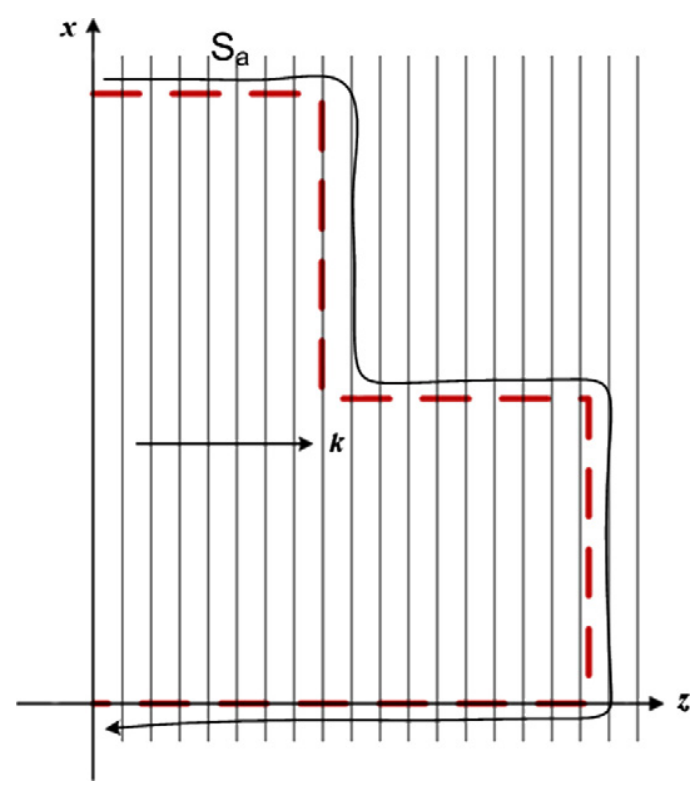

Fig. 3. (a) Real part of the propagating plane waves intersected by the $1 \mathrm{D}$ manifold $S_{a}$. (b) Corresponding propagating plane wave.

The matrix $\mathbf{R}$ is an upper triangular matrix. The inner product of $\boldsymbol{\psi}$ by the orthogonal basis functions will provide possible solutions for the complex coefficients of the plane waves

$\mathbf{Q}^{\mathrm{H}} \boldsymbol{\psi}=\mathbf{R} \mathbf{a}$.

Back-substitution can be used to calculate the elements of the vector $\mathbf{a}$, because $\mathbf{R}$ is an upper triangular matrix, but backsubstitution may not generally provide a robust reconstruction. Therefore, we use the singular value decomposition of $\mathbf{R}$ to obtain the complex amplitudes of the plane waves. Then, the linear relation between the diffraction field on the curved surface $S_{a}$ and the complex coefficients of the propagating plane waves can be expressed as

$\mathbf{Q}^{\mathrm{H}} \boldsymbol{\psi}=\mathbf{U} \mathbf{\Sigma} \mathbf{V}^{\mathrm{H}} \mathbf{a}$

where the column vectors of $\mathbf{U}$ are the eigenvectors of $\mathbf{R} \mathbf{R}^{\mathrm{H}}$ and the column vectors of $\mathbf{V}$ are the eigenvectors of $\mathbf{R}^{\mathrm{H}} \mathbf{R}$. The diagonal elements of the matrix $\Sigma$ are the eigenvalues of the matrix $\mathbf{R}$. A robust solution for the complex coefficients of the plane waves is obtained by discarding the singular values which are closer to the computational machine error. The diagonal elements of the diagonal matrix $\Sigma^{-1}$ can be obtained as $\frac{1}{l_{i}}$ where $l_{i}$ is the $i$ th diagonal element of the matrix $\mathbf{\Sigma}$. 


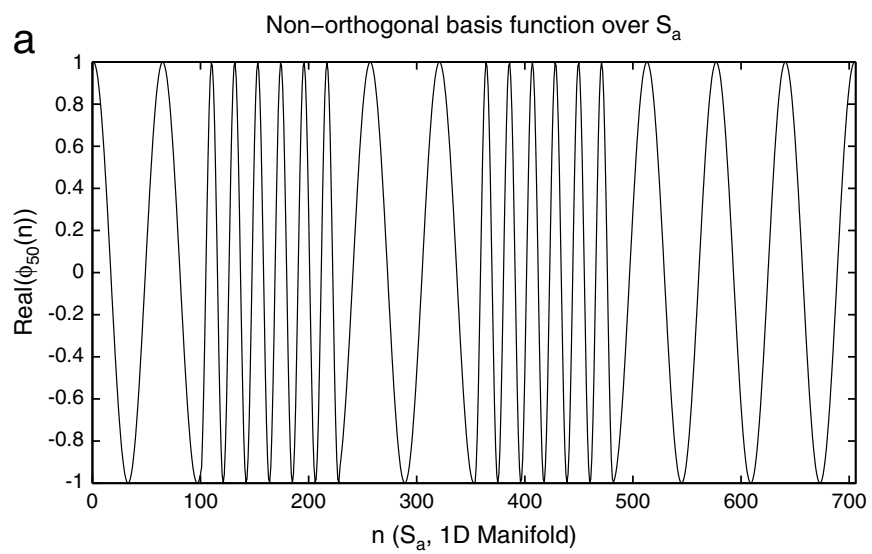

b

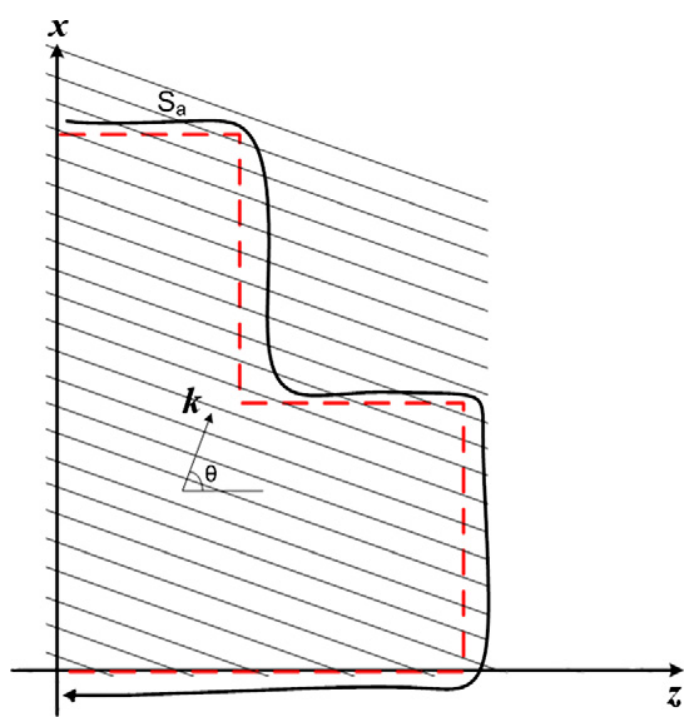

Fig. 4. (a) Real part of the propagating plane waves intersected by the $1 \mathrm{D}$ manifold $S_{a}$ (b) Corresponding propagating plane wave.
By eliminating the singular values which are close to the computational machine error, we prevent amplification of numerical errors. The compensated solution of the vector $\mathbf{a}$ is

$\mathbf{a}=\mathbf{V} \mathbf{\Sigma}^{-1} \mathbf{U}^{H} \mathbf{Q}^{H} \boldsymbol{\psi}$

The same approach can be easily extended to find the diffraction field over the entire 3D space arising from a field specified over a 2D curved surface. This is more realistic since most real life applications are $3 \mathrm{D}$. We again assume that the diffraction field over the 3D space can be expressed as the superposition of plane waves, and our initial aim is to obtain the complex amplitudes of these plane waves. The diffraction field over the 2D curved surface $S_{a}$ can be expressed in terms of the profiles of the plane waves on $S_{a}$. The same algorithm used in the 2D space case can be employed after vectorizing the 2D discrete field and coefficient arrays. Each plane wave profile on $S_{a}$ is stored as a 2D array:

$\boldsymbol{\psi}_{S_{a}}=\left[\begin{array}{llll}\boldsymbol{\Psi}_{{S_{1}}_{1}} \mid & \boldsymbol{\psi}_{S_{a_{2}}} \mid & \ldots & \mid \boldsymbol{\psi}_{S_{a_{N}}}\end{array}\right]$

By concatenating the $1 \mathrm{D}$ arrays, $\psi_{S_{a}}$, we can represent the 2D field over the curved surface $S_{a}$ as a 1D array $\psi_{v}$. Each column vector $\psi_{S_{a_{i}}}$ represents the variation of the 2D field over the curved surface $S_{a}$ along the $y$ coordinate and the index $i$ denotes variation along the $x$ coordinate. The obtained 1D array $\boldsymbol{\psi}_{v}$ is

$\boldsymbol{\psi}_{v}=\left[\begin{array}{c}\boldsymbol{\psi}_{S_{a_{1}}} \\ \boldsymbol{\Psi}_{S_{a_{2}}} \\ \vdots \\ \boldsymbol{\psi}_{S_{a_{N}}}\end{array}\right]$.

Naturally the 1D array $\boldsymbol{\psi}_{v}$ has a very large size.

In $3 \mathrm{D}$ representation, a which is the complex coefficients of the plane waves is a $2 \mathrm{D}$ array similar to $\psi_{S_{a}}$. Conversion of a into 1D array is also needed and the same method used for $\psi_{S_{a}}$ is employed.

The signal set which consists of the profiles of the plane waves on $S_{a}$ is expressed as $\Phi_{i, j}=\exp \left(j \mathbf{k}_{i, j}^{T} \mathbf{x}\right)$, where $\mathbf{k}_{i, j}=\left[k_{m_{i}}, k_{m_{j}}, \sqrt{k^{2}-k_{m_{i}}^{2}-k_{m_{j}}^{2}}\right]$ and $i j$ denote the indices of the discrete spatial frequencies along the $x$
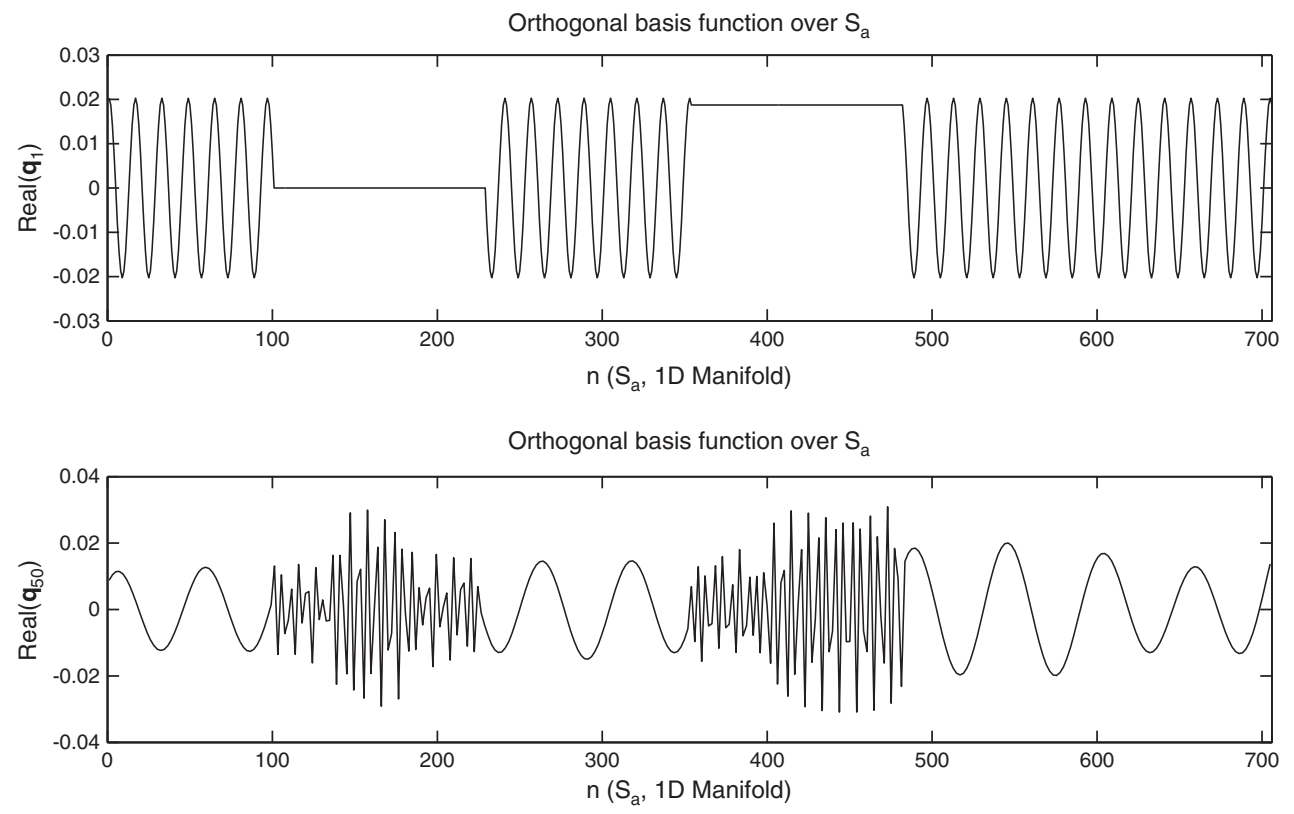

Fig. 5. Orthgonalized functions along the manifold $S_{a}$. 

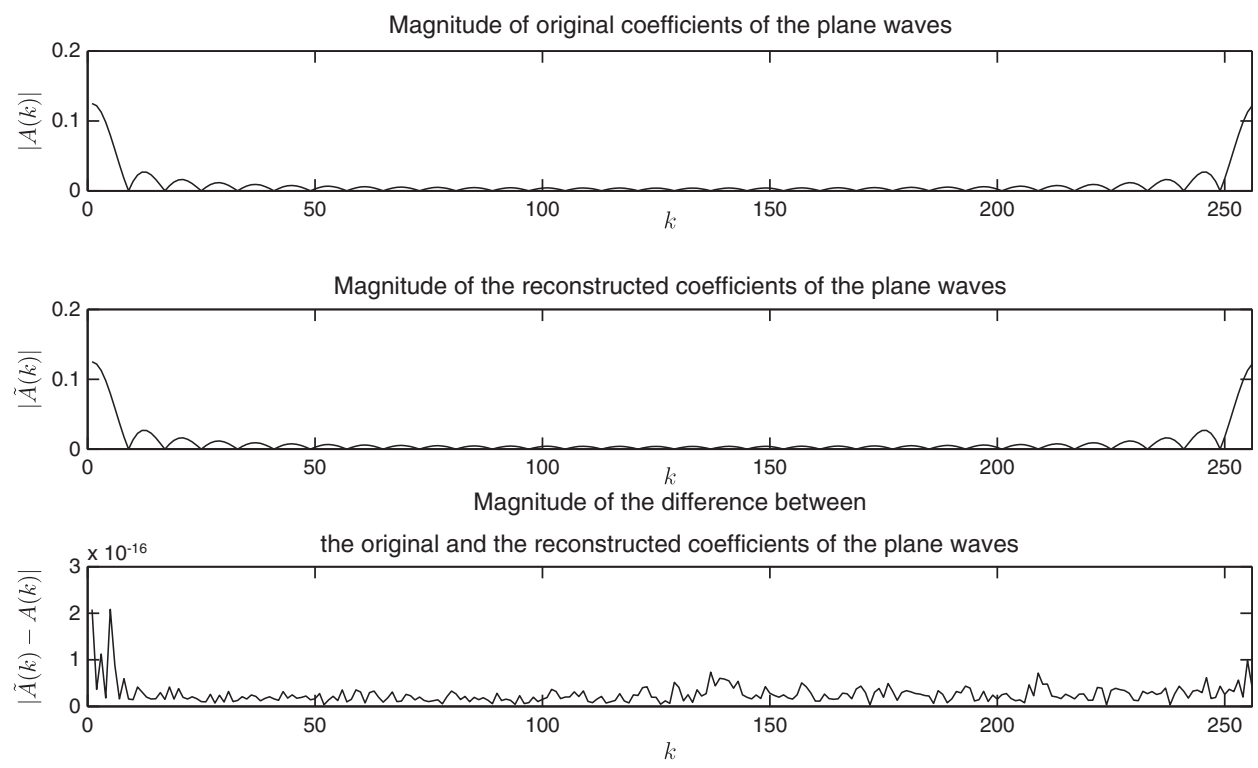

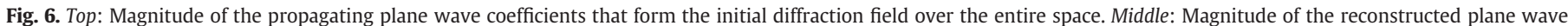
coefficients by Eq. (21). Bottom: Magnitude of the difference between the initial and the reconstructed plane wave coefficients.

and $y$ axes, respectively. The 2D array representations of these signals are obtained as

$\Phi_{i, j}=\left[\begin{array}{llll}\Phi_{i, j, 1} \mid & \Phi_{i, j, 2} \mid & \ldots & \mid \Phi_{i, j, N}\end{array}\right]$

where $\Phi_{i, j, l}$ is a column vector as in Eq. (22). Then, the 2D array is converted into a 1D array as in Eq. (23),

$\Phi_{v, i, j}=\left[\begin{array}{c}\Phi_{i, j, 1} \\ \Phi_{i, j, 2} \\ \vdots \\ \Phi_{i, j, N}\end{array}\right]$.
The system matrix $\Phi$ is now formed by the 1D arrays $\Phi_{v ; i, j}$ as

$\Phi=\left[\begin{array}{llllllllllllll}\Phi_{v ; 1,1} \mid & \ldots & \mid & \Phi_{v ; 1, N} \mid & \Phi_{v ; 2,1} \mid & \ldots & \mid & \Phi_{v ; 2, N} \mid & \mid & \Phi_{v ; N, 1} \mid & \ldots & \mid & \Phi_{v ; N, N}\end{array}\right]$.

Storage of these arrays requires the allocation of huge amounts of memory. As a consequence, applications of the presented algorithm to the $3 \mathrm{D}$ case may not be feasible for high resolution cases.

Therefore, the matrix equation that describes the calculation of 3D diffraction field can be expressed as

$\psi_{v}=\Phi \mathbf{a}$
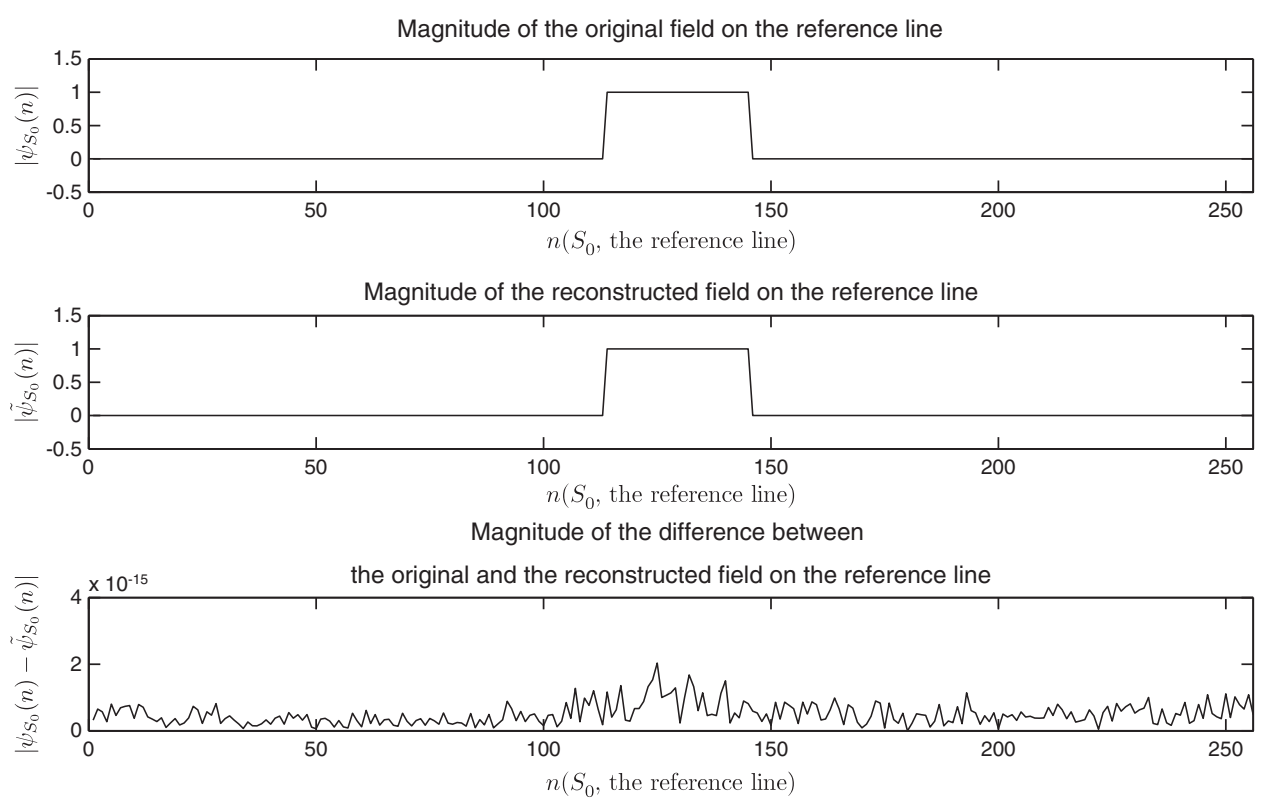

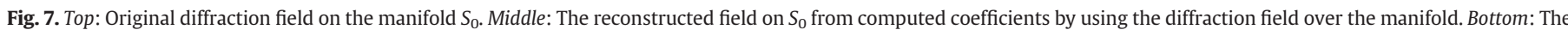
magnitude of the difference between the original and the reconstructed fields on $S_{0}$. 

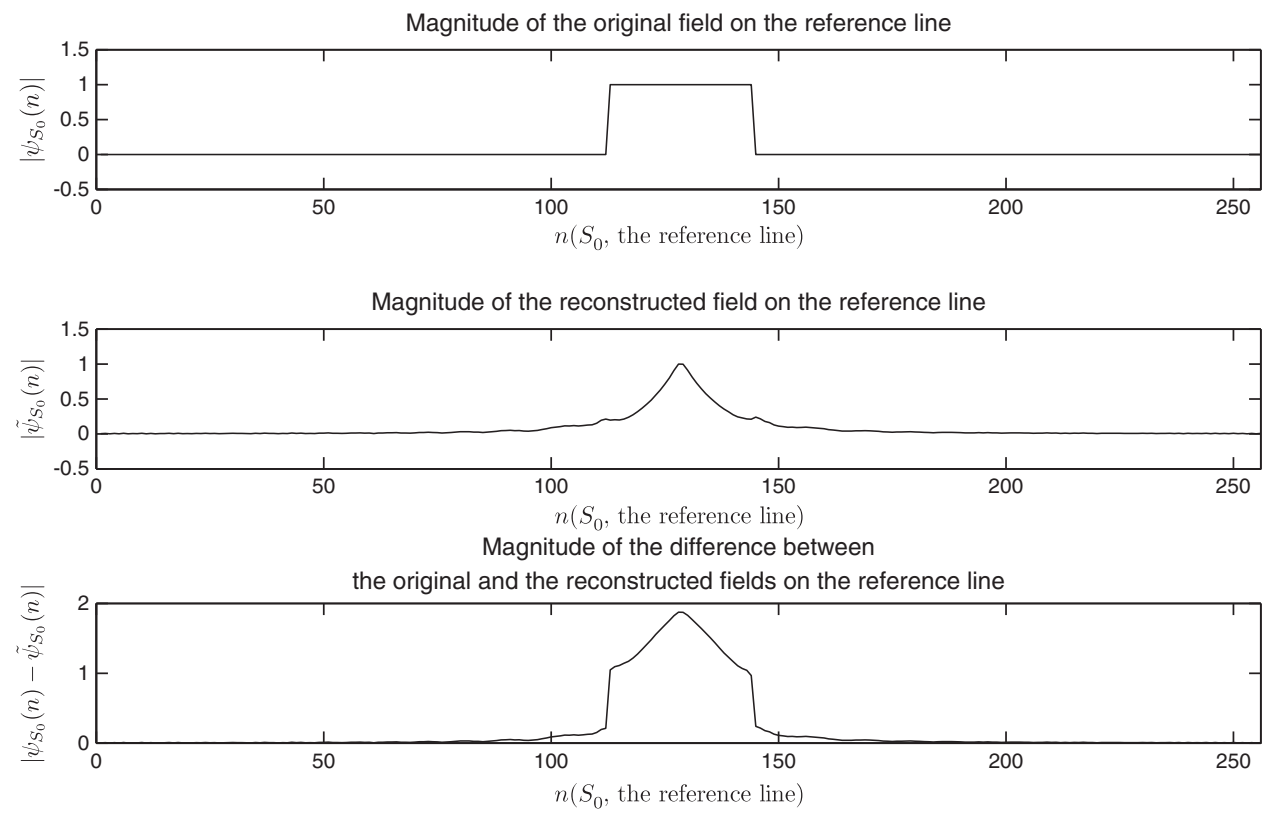

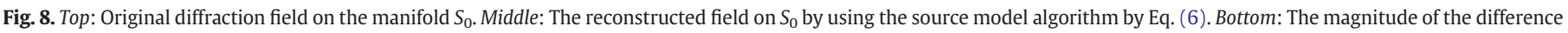
between the original and the reconstructed fields on $S_{0}$.

where $\mathbf{a}$ is a $1 \mathrm{D}$ array which denotes the complex coefficients of the propagating plane waves.

\section{Simulation results}

Here we illustrate and evaluate the proposed field model algorithm and compare it with the source model approach for both the 2D and 3D cases. We have tested the algorithms for several different fields and for several curved surfaces, of which two examples are shown here. The proposed field model algorithm provides perfect reconstruction of the field when the given information (the field values at specified sample points over the curved surface $S_{a}$ ) is sufficient.

In our first example, the diffraction field over 2D space arising from a $1 \mathrm{D}$ object is considered (Fig. 2).

The "curved" surface $S_{a}$ and the segment over the $x$-axis form a closed loop. We denote the sampling interval by $X$. The parameters $m$, $n, p, q$ that define the lengths of the segments that make up the manifold $S_{a}$ are chosen as $100,128,125$, and 128 , respectively. The number of samples along the $x$-axis is denoted by $N$, which we choose
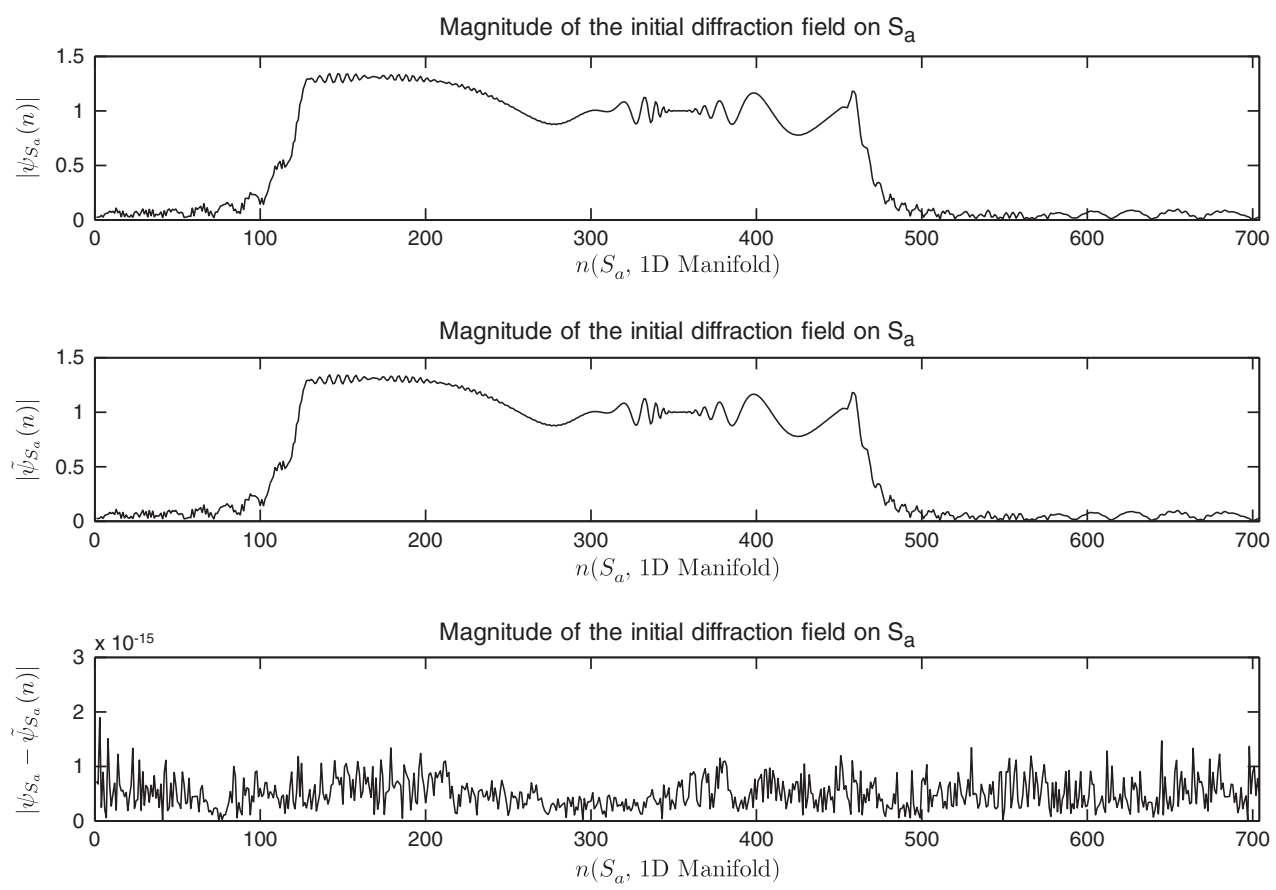

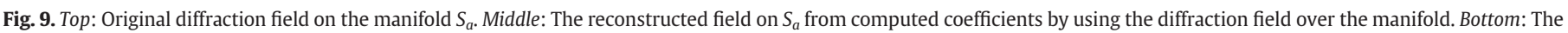
magnitude of the difference between the original and the reconstructed fields on $S_{a}$. 


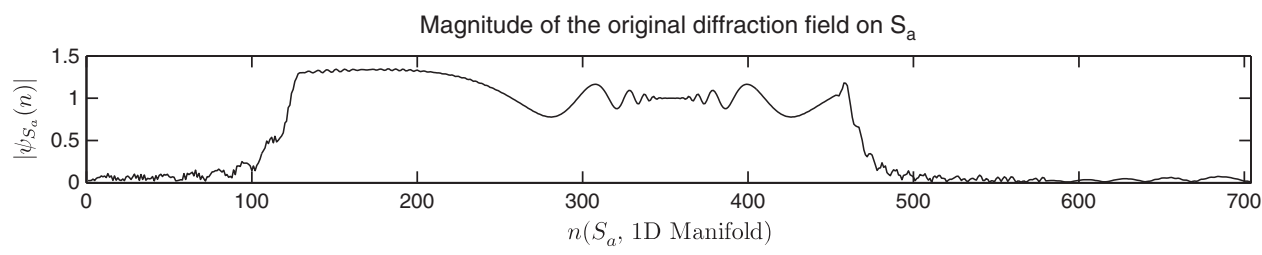

Magnitude of the reconstructed diffraction field on $\mathrm{S}_{\mathrm{a}}$
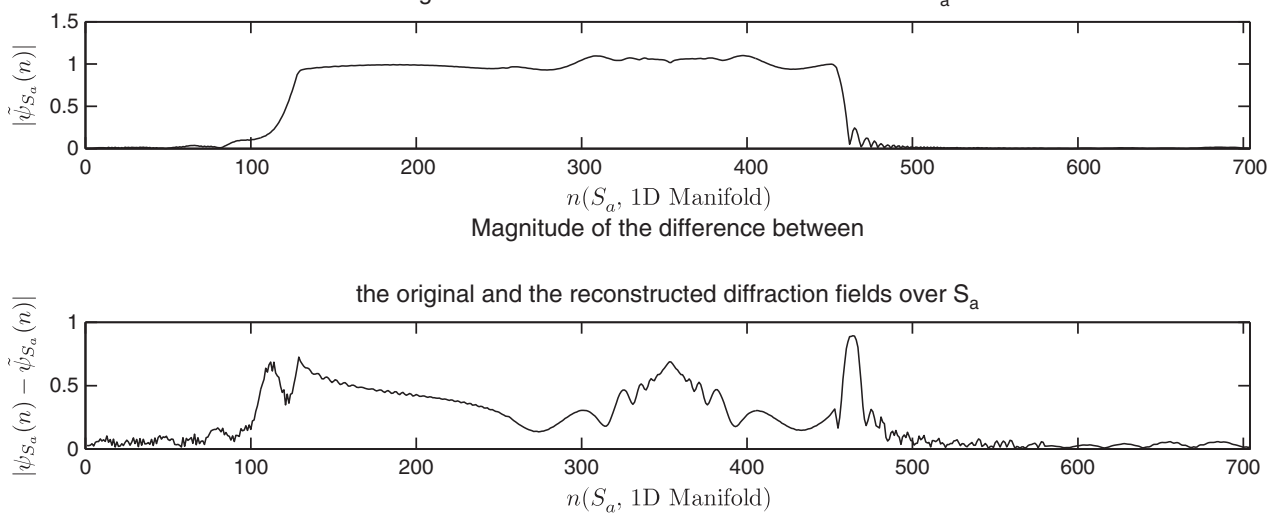

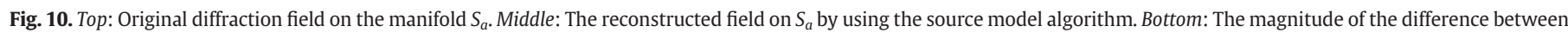
the original and the reconstructed fields on $S_{a}$.

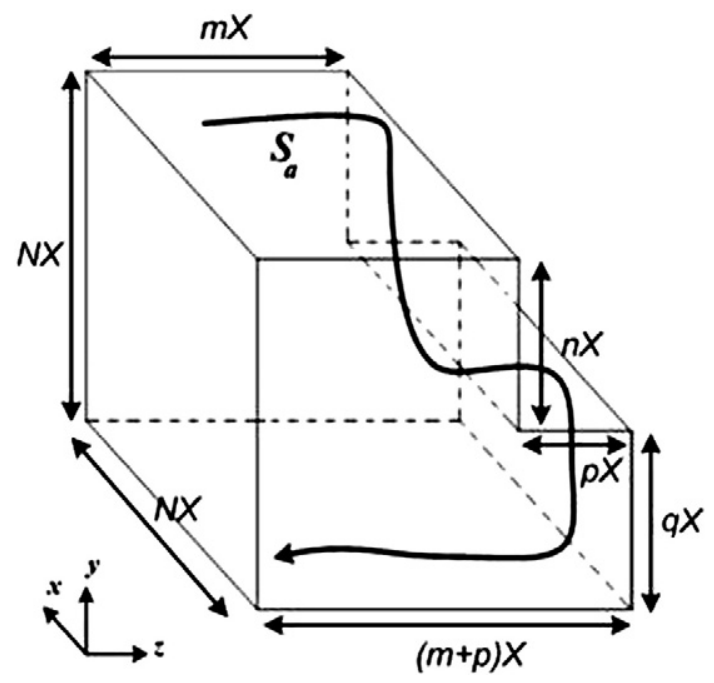

Fig. 11. Example where we wish to reconstruct the 3D diffraction field from the field given over the 2D curved surface $S_{a}$.

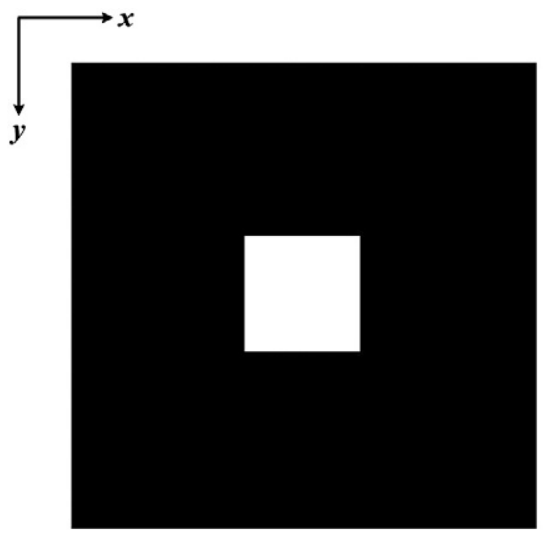

a as 256, and the variable $X$ is taken as $\lambda / 2$ where the wavelength $\lambda$ is chosen as $0.5 \mu \mathrm{m}$. Larger sampling step sizes can be used if the bandwidth is smaller than $\frac{1}{\lambda}$ cycles/m, which is the maximum possible spatial frequency along the $x$-axis for propagating waves. As mentioned in Section 3, the field over the entire space is composed of propagating plane waves only. The propagation of these plane waves is explained by Eq. (12) for the discrete case. We take the number of propagating plane waves also as 256. In Figs. 3(b) and 4(b), two of these 256 propagating plane waves are illustrated. The propagation directions of the plane waves can be found by the spatial frequencies $k_{m_{x}}=\frac{2 \pi}{N X} m_{x}$, where $m_{x} \in[-N / 2, N / 2)$. As a consequence of employing a finite number of propagating waves with discrete $k_{m_{x}}=\frac{2 \pi}{N X} m_{x}$ and integer $m_{x}$ which is defined as $m_{x} \in[-N / 2, N / 2)$, the entire $2 \mathrm{D}$ field is periodic along the $x$-direction with a period $N X$.

In order to assure zero error in the minimization of Eq. (13), the given field $\psi(\mathbf{x})$ on the manifold $S_{a}$ should be consistent with the wave equation. One way to assure a consistent $\psi(\mathbf{x})$ on $S_{a}$ for purposes of our numerical experiments is to start with some field pattern on the reference line, and then propagate it to $S_{a}$. Ensuring that the specified

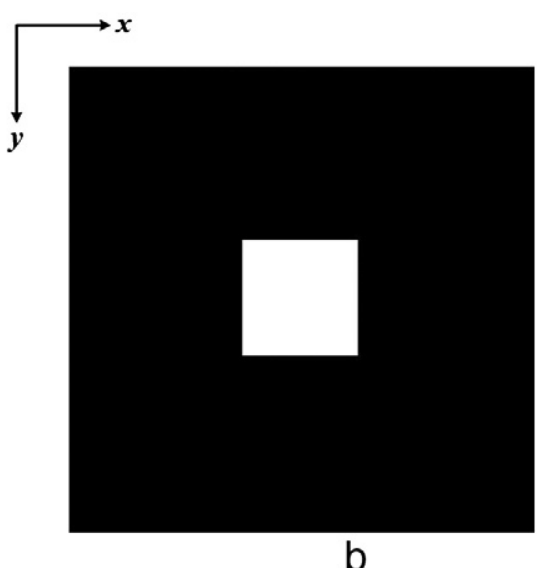

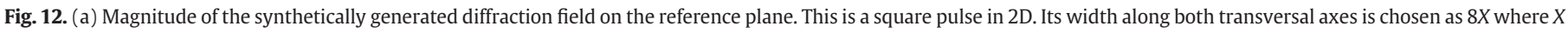

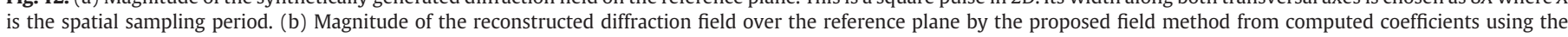
diffraction field over the manifold by Eq. (21). 


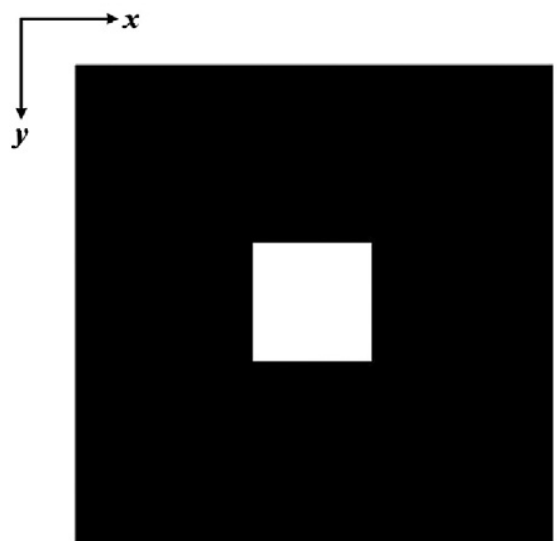

a

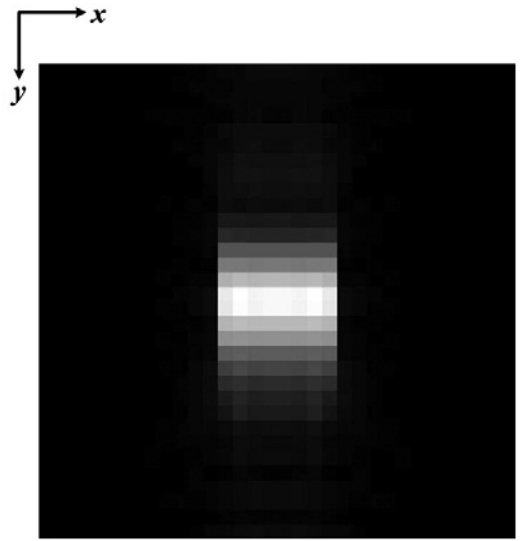

b

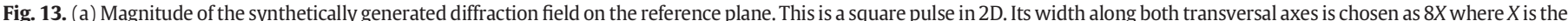

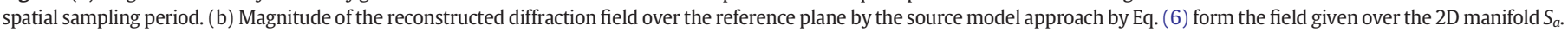

fields are consistent in our experiments, as they would be if the specified fields are indeed samples of a true propagating wave, we check whether our proposed method indeed can reconstruct the pattern on the reference line perfectly.

In our example, a synthetic signal on the reference line $z=0$ is chosen. From this we compute the diffraction field over the entire space. The synthetic signal on the reference line is chosen as a unit square pulse whose width is $\frac{1}{8}$ of $N X$. Then, the complex amplitudes of the propagating planes can be obtained from the synthetic signal by taking the forward Fourier transform. By using the propagation behavior of the plane waves through the free space the diffraction field over the entire space can be computed. These plane waves are

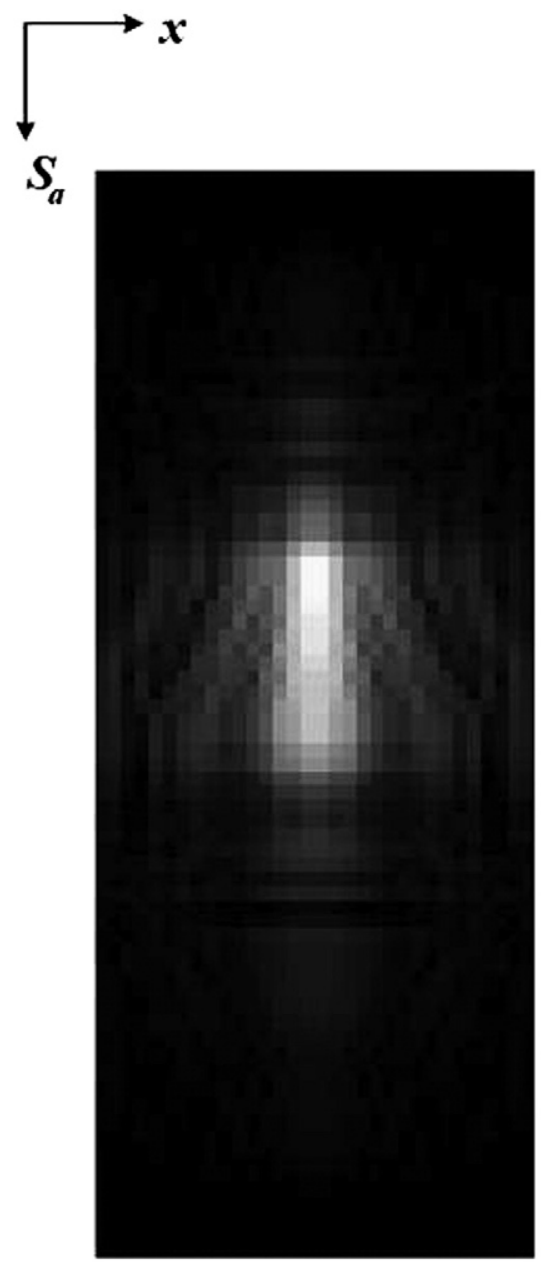

a

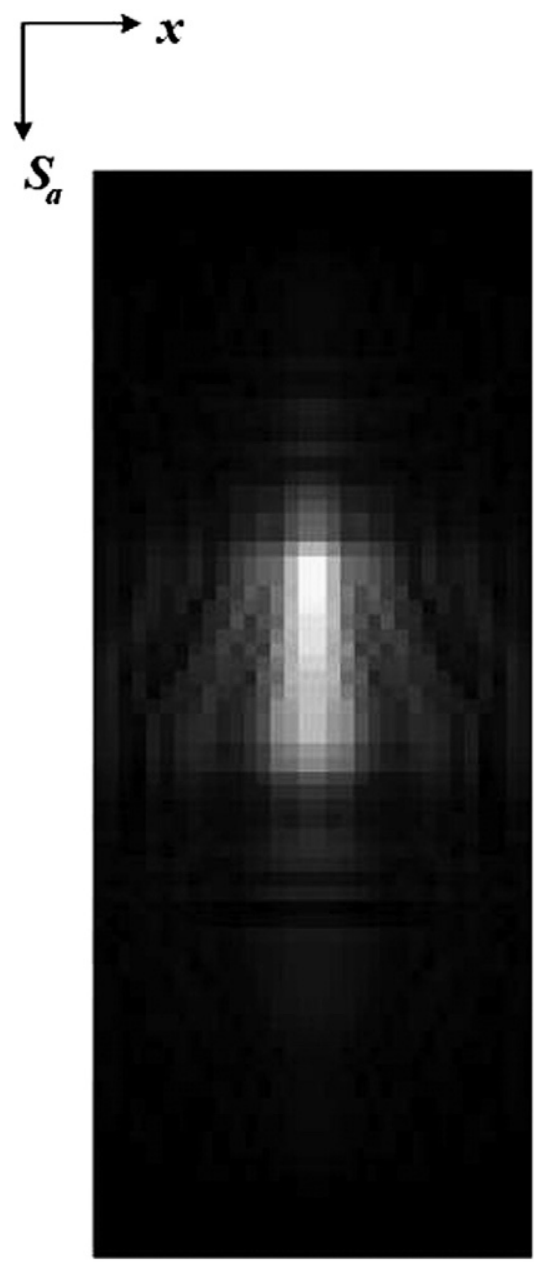

b

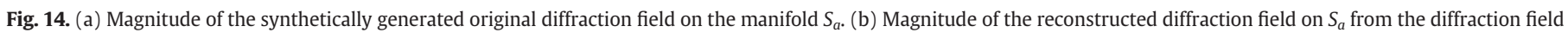
over the reference plane that was computed by the proposed field method. 
intersected by the manifold $S_{a}$. The signals obtained by the intersection are expressed as $\Phi_{i}$ where $i$ denotes the spatial frequency $k_{m_{x}}$. Real parts of $\Phi_{1}$ and $\Phi_{50}$, the cross-sections of the plane waves along the manifold $S_{a}$, can be seen in Figs. 3 and 4, respectively.

After performing Gram-Schmidt orthogonalization of these plane waves along the manifold as in Eq. (17), we obtain a set of orthogonal functions $\mathbf{q}_{i}$ where $i$ is related to spatial frequency as in $\Phi_{i}$. Real parts of the orthogonal basis functions that are related to the functions given in Figs. 3 and 4, are shown in Fig. 5.

Then, the complex coefficients of the plane waves are obtained by Eq. (21). In Fig. 6, the magnitudes of the reconstructed coefficients of the plane waves that form the diffraction field over the space are illustrated. The magnitudes of the differences between the initial and the reconstructed coefficients are also shown in Fig. 6.

Both Figures represent discrete values for $k_{m_{x}}=\frac{2 \pi}{N X} m_{x}$ where $m_{x}$ is an integer such that $m_{x} \in[-N / 2, N / 2)$, corresponding to plane wave components of the diffraction field, but figures plotted as continuous graphs by using linear interpolation for convenience. Then, the diffraction field along the reference line is reconstructed from the intersections of the plane waves along the reference line. The original and the reconstructed fields are shown in Fig. 7. The magnitude of the difference between the original and the reconstructed fields is also given in Fig. 7.

To compare the performance of a source model algorithm with the proposed field model algorithm, the same scenario shown in Fig. 2 is implemented by using the source model algorithm given by Eq. (6). The magnitude of the reconstructed and the original fields along the reference line can be seen in Fig. 8. The difference between the original and the reconstructed fields is quite significant.

Another example is presented in Figs. 9 and 10. First we compute the field on the reference line from the given field over the curved surface. Then we compute the diffraction field along the manifold $S_{a}$ from the found field over the reference line, for comparison. Such reconstructed field on $S_{a}$ and its deviation from the original are shown. The results for the proposed accurate field model are shown in Fig. 9. On the other hand, the results for the commonly used source model are presented in Fig. 10. The source model does not yield the accurate results obtained by the field model.

As noted, the application of the proposed method to 3D fields requires large amounts of memory; nevertheless we present a simple example as a proof of concept. The reference plane is assumed to have 32 samples along both transversal axes. The $32 \times 32$ synthetic signal on the reference plane is assumed to have a unit magnitude 2D square pulse located at its center, with a width of 8 samples along both transversal dimensions. An illustration of the chosen field over the reference plane can be seen in Fig. 12(a). Then, the diffraction field over the entire 3D space due to this 2D square pulse is computed by using the plane wave decomposition. The 2D curved surface employed in the 3D simulations is shown in Fig. 11.

The parameters $m, n, p, q$ are taken as $12,16,10$, and 16 , respectively and $N=32$. The spatial sampling interval is chosen as $\frac{\lambda}{2}$ and the optical wavelength is chosen as $0.5 \mu \mathrm{m}$ as in the $2 \mathrm{D}$ space example discussed earlier. To have a consistent field, we start by setting the field on the manifold as the intersection of a 3D field by the

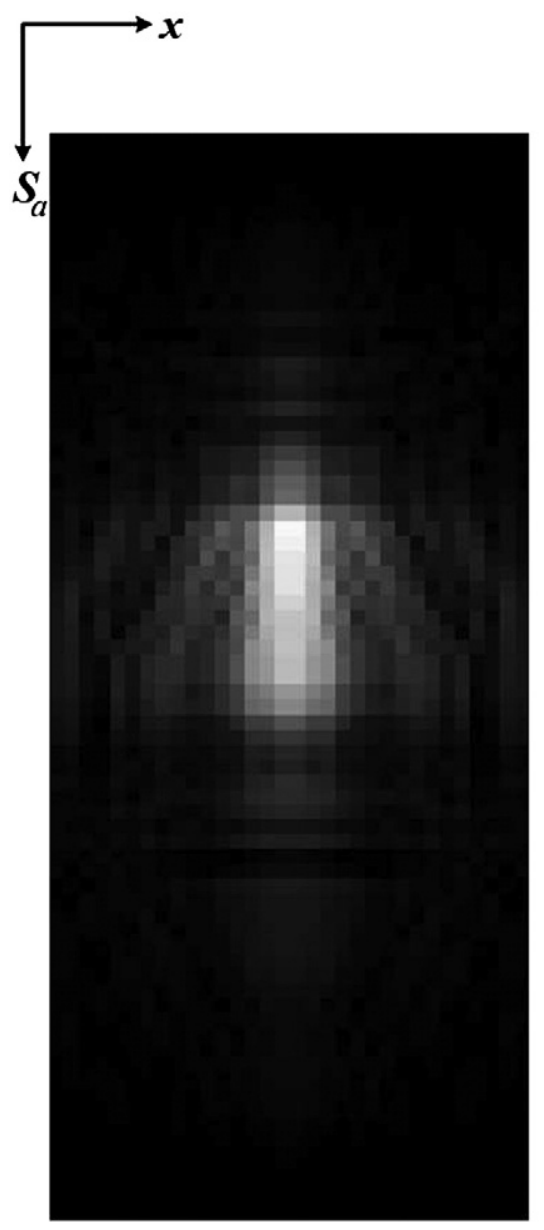

a

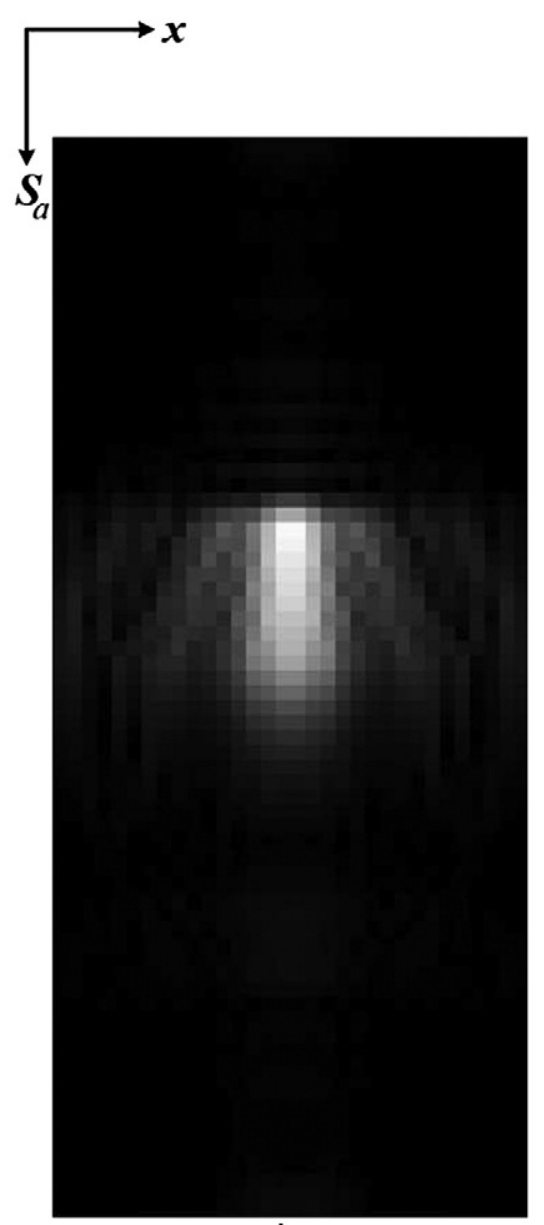

b

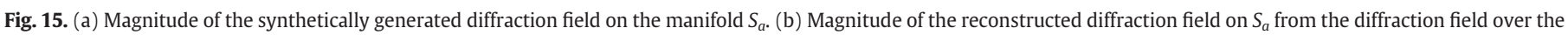
reference plane that was computed by the source model approach. 
manifold. Such a field over the 2D manifold $S_{a}$ is shown in Fig. 14. By using the proposed algorithm as outlined by Eqs. (21-26), the diffraction field over the reference plane, due to the given field over the 2D manifold $S_{a}$, is computed. The difference between the initial and the reconstructed fields on the reference plane determines the performance of the proposed algorithm. The reconstructed field over the reference plane is shown in Fig. 12b.

The same 3D space simulation is performed by source model approach of Eq. (6) and the obtained results are shown in Fig. 13(b). The original and the reconstructed fields on the reference plane are shown in Fig. 13. As seen in this figure, the source model does not provide correct results.

The diffraction field over the manifold $S_{a}$ is computed from the reconstructed field over the reference plane that was obtained by the proposed field model. Again a perfect result is obtained. The reconstructed diffraction field over the 2D manifold $S_{a}$ can seen in Fig. 14.

For comparison, the reconstructed field over the 2D manifold $S_{a}$ from the diffraction field on the reference plane that was computed by a source model approach, is given in Fig. 15(b).

\section{Conclusion}

Computation of the exact diffraction field over the space from the field specified on an arbitrary curved surface is a basic problem with several applications. Our interest in this problem stems from computer generated holography where computation of the diffraction field from an object with arbitrary surface profile is necessary to obtain the optical field which must be generated at the display end of the system.

Commonly, the calculation of the diffraction field over the space from field values on a curved surface is based on what we referred to as the source model. However, these approaches are only approximate and provide satisfactory results only under restricted conditions, such as planar objects or objects which have surfaces that only slightly deviate from a plane [4]. On the other hand, field model based algorithms provide computation of the exact diffraction field over the entire space, and errors arise only due to arithmetic precision. In this work, we present a field model algorithm based on decomposition of the given field on a curved surface onto a set of functions which are profiles of the propagating plane waves on the curved surface. In practice, the presented approach can be easily used for 2D problems with realistic resolutions. However, its applications to 3D problems demand high computational complexity, and therefore, realistic size problems cannot be accommodated with presently available computers. Although the presented solution is computationally costly, we are not aware of any other method that yields exact results for the given problem.

\section{Acknowledgement}

H. M. Ozaktas acknowledges partial support of the Turkish Academy of Sciences.

\section{Appendix A}

Here we show that the profiles of the plane waves on the curved surface $S_{a}$ are not orthogonal through a simple example (Fig. 16).

The inner product of two different complex exponentials over $S_{a}$ can be expressed as

$$
\left.\left\langle\exp \left(j \mathbf{k}_{1}^{T} \mathbf{x}\right), \exp \left(j \mathbf{k}_{2}^{T} \mathbf{x}\right)\right\rangle\right|_{S a}=\int_{S_{a}} \exp \left[j\left(\mathbf{k}_{1}-\mathbf{k}_{2}\right)^{T} \mathbf{x}\right] d S
$$

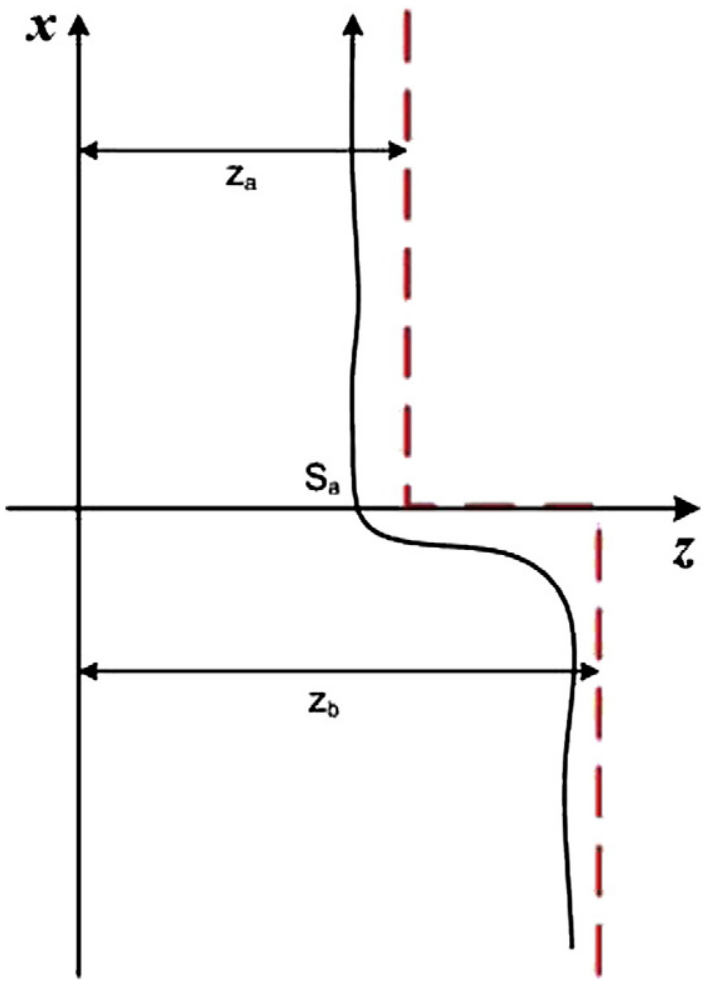

Fig. 16. A simple 1D example to illustrate the nonorthogonality of the profiles of the plane waves on $S_{a}$

$$
\begin{aligned}
= & \int_{S_{a}} \exp \left(j \mathbf{k}_{3}^{T} \mathbf{x}\right) d S \\
= & \int_{-\infty}^{0} \exp \left(j k_{3, x} x\right) \exp \left(j k_{3, z} z_{b}\right) d x+\int_{z_{b}}^{z_{a}} \exp \left(j k_{3, z} z\right) d z \\
& +\int_{0}^{\infty} \exp \left(j k_{3, x} x\right) \exp \left(j k_{3, z} z_{a}\right) d x \\
= & \left(-\frac{1}{j k_{3, x}}+\pi \delta\left(k_{3, x}\right)\right) \exp \left(j k_{3, z} z_{b}\right)+\frac{\exp \left(j k_{3, z} z_{a}\right)-\exp \left(j k_{3, z} z_{b}\right)}{j k_{3, z}} \\
& +\left(\frac{1}{j k_{3, x}}+\pi \delta\left(k_{3, x}\right)\right) \exp \left(j k_{3, z} z_{a}\right)
\end{aligned}
$$

Since, the result of the integral given above is not always proportional to $\delta\left(k_{3, x}\right)$, the profiles of the planes waves on the curved surface $S_{a}$ are not in general orthogonal. The first and the third integrals in Eq. (30) are computed by using the Fourier transform of the sign function sgn $(x)$ :

$\operatorname{sgn}(x)=\left\{\begin{array}{cc}\frac{1}{2} & x \geq 0 \\ -\frac{1}{2} & x<0\end{array}\right.$.

The result obtained in Eq. (31) shows that orthogonality may not be satisfied even for this simple example. Therefore, to obtain orthogonal functions, we employ an orthogonalization as discussed in Section 4. 


\section{References}

[1] H.M. Ozaktas, L. Onural, Springer Series in Signals and Communication Technology, 2008.

[2] http://www.3dtv-research.org.

[3] G.B. Esmer, V. Uzunov, L. Onural, H.M. Ozaktas, A. Gotchev, Signal Process. Image Commun. 22 (2007) 178.

[4] G.B. Esmer, L. Onural, H.M. Ozaktas, V. Uzunov, A. Gotchev, 3DTV-ConII 2008, Istanbul, Turkey, 2008.

[5] M. Lucente, Diffraction-specific fringe computation for electro-holography, Ph.D. Dissertation, Massachusetts Institute of Technology, Cambridge, MA USA, 1994.

[6] J.W. Goodman, Introduction to Fourier Optics, Mc-Graw-Hill, New York, 1996.

[7] M. Born, E. Wolf, Principles of Optics: Electromagnetic theory of propagation, interference and diffraction of light, Cambridge University Press, New York, 1980.

[8] H. Kang, T. Yamaguchi, H. Yoshikawa, S.-C. Kim, E.-S. Kim, Appl. Opt. 47 (31) (2008).

[9] C. Petz, M. Magnor, Proc. SPIE, Practical Holography XVII and Holographic Materials IX, vol. 5005, 2003, p. 266.

[10] H.M. Ozaktas, Z. Zalevsky, M.A. Kutay, Series in Pure and Applied Optics, John Wiley and Sons, 2001.

[11] L. Onural, H.M. Ozaktas, Signal Process. Image Commun. 22 (2007) 169.

[12] B.E.A. Saleh, M.C. Teich, Fundamentals of Photonics, John Wiley and Sons, Inc., 1991.
[13] R. Ziegler, P. Kaufmann, M. Gross, IEEE Trans. Visual. Comput. Graphics 13 (2007) 403.

[14] M. Janda, I. Hanák, L. Onural, J. Opt. Soc. Am. A 25 (12) (2008) 3083.

[15] S. Cunningham, Computer Graphics: Programming in OpenGL for Visual Communication, Prentice Hall, 2006.

[16] G.B. Esmer, Calculation Of Scalar Optical Diffraction Field From Its Distributed Samples Over The Space, Ph.D. Dissertation, Bilkent University, Dept. of Electrical and Electronics Engineering, Ankara, Turkey, 2010.

[17] L. Onural, A. Gotchev, H.M. Ozaktas, E. Stoykova, IEEE Trans. Circuits Syst. Video Technol. 17 (11) (2007) 1631.

[18] G.C. Sherman, J. Opt. Soc. Am. 57 (1967) 546.

[19] É. Lalor, J. Opt. Soc. Am. 58 (1968) 1235

[20] V. Uzunov, A. Gotchev, G.B. Esmer, L. Onural, H. Ozaktas, TICSP Series 34, Florence Italy, 2006.

[21] E. Ulusoy, G.B. Esmer, H.M. Ozaktas, L. Onural, A. Gotchev, V. Uzunov, ICIP 2006, Atlanta, GA, USA, 2006.

[22] G.B. Esmer, L. Onural, V. Uzunov, A. Gotchev, H.M. Ozaktas, 3DTV-Con 2007, Kos Island, Greece, 2007.

[23] M. Kovachev, R. Ilieva, P. Benzie, G.B. Esmer, L. Onural, J. Watson, T. Reyhan, ThreeDimensional Television: Capture, Transmission, Display, Chapter 15: Holographic 3DTV Displays Using Spatial Light Modulators, Springer-Verlag, Berlin Heidelberg, 2008 